\title{
SURVIVAL-PAIRS OF COMMUTATIVE RINGS HAVE THE LYING-OVER PROPERTY
}

\author{
Jim Coykendall ${ }^{1}$ and David E. Dobbs ${ }^{2}$ \\ ${ }^{1}$ Department of Mathematics \\ North Dakota State University \\ Fargo, ND. 58105-5075 \\ E-mail: jim.coykendall@ndsu.nodak.edu \\ ${ }^{2}$ Department of Mathematics \\ University of Tennessee \\ Knoxville, TN. 37996 \\ E-mail: dobbs@math.utk.edu
}

July 16, 2003

\begin{abstract}
Let $R \subseteq T$ be a unital extension of commutative rings. It is proved that $(R, T)$ is a lying-over pair (in the sense that $A \subseteq B$ satisfies LO for all rings $R \subseteq A \subseteq B \subseteq T$ ) if (and only if) $(R, T)$ is a survival-pair (in the sense that $\mathcal{P} T \neq T$ for all rings $R \subseteq A \subseteq T$ and all prime ideals $\mathcal{P}$ of $A$ ). As a consequence, $T$ is integral over $R$ if (and only if) $(R[X], T[X])$ is a survival-pair, where $X$ is an indeterminate over $T$. It is also proved that a unital homomorphism of commutative rings satisfies LO if and only if it is universally a survival homomorphism.
\end{abstract}

2000 Mathematics Subject Classification.

Primary 13B24, 13B21, 13B99;

Secondary 13A15, 13G05, 13B25, 14A15.

\section{INTRODUCTION}

All rings considered are commutative with identity; all inclusions of rings and all ring homomorphisms are unital. As in [6], if $\wp$ is a property of some ring extensions, we say that $(R, T)$ is a $\wp$-pair in case $R \subseteq T$ are rings such that $A \subseteq B$ satisfies $\wp$ for all rings $R \subseteq A \subseteq B \subseteq T$. The "pair" approach figures in the result that helped to motivate both [5] and [6], namely, [6, Folklore Theorem, p. 454]: a ring extension $R \subseteq T$ is integral if and only if $(R, T)$ is 
both an INC-pair and an LO-pair. (Following [12, p. 28], we let INC, LO, and GU denote the incomparable, lying-over, and going-up properties, respectively. In particular, a ring extension $A \subseteq B$ satisfies LO if and only if each prime ideal $P$ of $A$ is of the form $Q \bigcap A$ for at least one prime ideal $Q$ of $B$.) Over the years, INC-pairs have become well-understood. They were introduced, without the terminology, in [5, Theorem, p. 38], which implies that $(R, T)$ is an INC-pair if and only if $R \subseteq T$ are rings such that each element of $T$ is a root of a polynomial over $R$ having a unit coefficient. In other words, we have [5, Corollary 4]: $(R, T)$ is an INC-pair if and only if $R \subseteq T$ is a P-extension (in the sense of GilmerHoffmann [10]). In addition, although the implication INC $\Longrightarrow$ MINC is nonreversible, the concepts of INC-pair and MINC-pair are equivalent [6, Example 2.2, Corollary 2.4(bis)]. More recently, Ayache-Jaballah [1] showed that INCpairs are the same as the residually algebraic pairs. For a survey of current knowledge about INC-pairs, see [9, Section 6.5]. The present paper contributes to deepening our understanding of LO-pairs.

LO-pairs were introduced in [6]. Subsequent work on this topic has included a study of LO-pairs of affine algebras [13] and a recent non-integral example [2] that answered a question raised in [6, Remark 3.12(b)]. Much of [6] was motivated by the following non-reversible implications connecting properties of ring extensions: $\mathrm{GU} \Longrightarrow \mathrm{LO} \Longrightarrow$ survival. (Following [12, p. 35], we say that if $R \subseteq T$ are rings and $I$ is a proper ideal of $R$, then $I$ survives in $T$ if $I T \neq T$, that is, if $1 \notin I T$; and that $R \subseteq T$ is a survival extension if each proper (resp., prime) ideal of $R$ survives in T.) In the spirit of [6, Corollary 2.4(bis)], it was shown in [6, Corollary 3.2] that LO-pairs are the same as GU-pairs. It seems irresistible to ask if they are also the same as the survival-pairs. Our main result, Theorem 2.2 , settles the matter.

Although each LO-pair must be a survival-pair, the converse does not seem obvious to us. In fact, although the property of being an LO-pair is easily seen to be a local property [6, Lemma 2.11(c)], we do not consider it to be obvious whether "survival-pair" is a local property. Nevertheless, there has long been compelling evidence suggesting that survival-pairs are the same as LO-pairs. For instance, a number of contexts have been identified in which the "survival-pair" and "LO-pair" concepts coincide: cf. [6, Theorem 2.7, Remark 2.8(a),(b),(c),(e)]. Another similarity of behavior arises from the fact that "survival-pair" plays the same role as "LO-pair" in sharpenings of the "Folklore Theorem" characterizing integrality [6, Theorem 2.1]. Similar phenomena occur in a "nearly integral" context [7, Theorem 2.14, Proposition 2.16] and in a result concerning complete integral closure [8, Proposition 2.1]. In our deepest result, Theorem 2.2, we give the underlying reason: survival-pairs are the same as LO-pairs.

In a number of contexts mentioned above (such as in [6, Theorem 2.7, Remark 2.8(a),(b),(c)]), the "survival-pair" condition is strong enough to imply integrality. Given the above-mentioned work in [5, Theorem, p. 38] and [1] on algebraic aspects of INC-pairs, it thus seems natural to pursue deeper connections between the "survival-pair" and "integrality" concepts. In this regard, Corollary 2.3 gives a new way in which the "survival-pair" concept can be used to characterize an integral ring extension. Moreover, Proposition 2.4 identifies a 
sense in which arbitrary survival-pairs (that is, LO-pairs) are "closer" to being integral than are arbitrary INC-pairs.

Moving beyond the context of ring extensions to that of ring homomorphisms, recall for motivation that integrality and LO are universal properties (in the sense of [11], namely, being preserved by arbitrary base change) and that "universally GU" is equivalent to integrality. It seems natural to ask if/how these facts relate to the natural generalizations of the "survival" and "survivalpair" concepts to ring homomorphisms. The answers are given in Proposition 2.6(c) and Theorem 2.7: a ring homomorphism is integral (resp., satisfies LO) if and only if it is universally a survival-pair homomorphism (resp., universally a survival homomorphism). While these results frankly do not seem as deep to us as Theorem 2.2, they are pleasantly complete, they serve to place the "survival"-related concepts into a more appropriate categorical setting, and we would hope that readers find them of potential use in other studies.

We next describe notational conventions. If $A$ is a ring, then $\operatorname{Spec}(A)$ denotes the set of prime ideals of $A ; U(A)$ denotes the set of units of $A$; and $J(A)$ denotes the Jacobson radical of $A$. If $f$ is a ring homomorphism, then $\operatorname{ker}(f)$ denotes the kernel of $f$. By an overring of an integral domain $R$, we mean any ring contained between $R$ and its quotient field. Finally $\subset$ denotes proper containment, and $X$ denotes an indeterminate over the appropriate coefficient ring. Any unexplained material is standard, as in [12].

\section{RESULTS}

We begin by collecting some facts that will be useful in the proof of our main result.

Lemma 2.1. Let $(R, T)$ be a survival-pair. Then:

(a) If $J$ is an ideal of $T$ and $I:=J \bigcap R$, then $(R / I, T / J)$ is a survival-pair.

(b) If, in addition, $R$ is integrally closed in $T$, then $U(R)=U(T)$.

Proof: (a) We show that $A \subseteq T / J$ is a survival extension for each ring $A$ between $R / I$ and $T / J$. Identifying $R / I=(R+J) / J$, we see that $A=S / J$ for some ring $S$ between $R+J$ and $T$. If survival fails for $A \subseteq T / J$, then $1 \in Q(T / J)$ for some $Q \in \operatorname{Spec}(A)$. Write $Q=q / J$ with $q \in \operatorname{Spec}(S)$. Then $1 \in q T+J=q T$, contradicting that $S \subseteq T$ is a survival extension.

(b) Deny. Choose $v \in U(T) \backslash R$. As $1 \in v T=v R[v] T$ and $R[v] \subseteq T$ is a survival extension, it follows that $1 \in v R[v]$, whence $v^{-1} \in R[v]$ and $v^{-1}$ is integral over $R$ [12, Theorem 15]. Since $v^{-1} \in T$ and $R$ is integrally closed in $T$, we now have that $v^{-1} \in R$. As $1=v^{-1} v \in R v^{-1} T$ and $R \subseteq T$ is a survival extension, $R v^{-1}=R$; that is, $v^{-1} \in U(R)$. Then $v=\left(v^{-1}\right)^{-1} \in R$, the desired contradiction. $\diamond$

We next present our main result.

Theorem 2.2. Let $R \subseteq T$ be rings. Then the following conditions are equivalent:

(1) $A \subseteq A[u]$ is a survival extension for all rings $A$ such that $R \subseteq A \subseteq T$ and all elements $u \in T$; 
(2) $A \subseteq A[u]$ satisfies $L O$ for all rings $A$ such that $R \subseteq A \subseteq T$ and all elements $u \in T$;

(3) $A \subseteq A[u]$ satisfies $G U$ for all rings $A$ such that $R \subseteq A \subseteq T$ and all elements $u \in T$;

(4) $(R, T)$ is a survival-pair;

(5) $(R, T)$ is an LO-pair;

(6) $(R, T)$ is a GU-pair.

Proof: It was shown in [6, Corollary 3.2] that conditions (5) and (6) are equivalent. Moreover, (6) implies (3) trivially, by the definition of $\wp$-pair. Also, (3) implies (2), since GU implies LO for extensions; and (2) implies (1) since LO implies survival for extensions. It remains to prove that (1) implies (4) and that (4) implies (5).

$(1) \Longrightarrow(4)$ : We give an indirect proof in the spirit of the proof of [6, Proposition 2.10]. Suppose that (1) holds but (4) fails. Then $A \subseteq B$ is not a survival extension, for some rings $R \subseteq A \subseteq B \subseteq T$. Pick $\mathcal{P} \in \operatorname{Spec}(\bar{A})$ such that $1 \in \mathcal{P} B$. Now consider the set

$$
\mathcal{S}:=\{D \mid D \text { is a ring, } A \subseteq D \subseteq B \text {, and } \mathcal{P} \text { survives in } D\},
$$

partially ordered by inclusion. Evidently, $\mathcal{S} \neq \emptyset$, for $A \in \mathcal{S}$. If $\left\{E_{\alpha}\right\}$ is a chain in $\mathcal{S}$, then $\bigcup E_{\alpha} \in \mathcal{S}$. (Indeed, if $1=\sum_{i=1}^{n} p_{i} e_{i}$ with $p_{i} \in \mathcal{P}, e_{i} \in E_{\alpha_{i}}$ and $E_{\alpha_{1}} \subseteq E_{\alpha_{2}} \subseteq \cdots \subseteq E_{\alpha_{n}}$, then $1 \in \mathcal{P} E_{\alpha_{n}}$, contradicting that $E_{\alpha_{n}} \in \mathcal{S}$.) Thus, Zorn's Lemma applies, producing a maximal element $A_{0}$ of $\mathcal{S}$. Since $\mathcal{P}$ does not survive in $B$, we have that $A_{0} \neq B$. Choose $u \in B \backslash A_{0}$. As $1 \notin \mathcal{P} A_{0}$, (1) ensures that $\mathcal{P} A_{0}$ survives in $A_{0}[u]$; that is, $1 \notin \mathcal{P} A_{0}[u]$. Thus $A_{0}[u] \in \mathcal{S}$, contradicting that $A_{0}$ is maximal in $\mathcal{S}$.

$(4) \Longrightarrow(5)$ : It suffices to prove that if $(R, T)$ is a survival-pair, then $R \subseteq$ $T$ satisfies LO. Deny. Fix $\mathcal{P} \in \operatorname{Spec}(R)$ such that no prime ideal of $T$ lies over $\mathcal{P}$. By [12, Theorem 10], $\mathcal{P}$ contains a minimal prime ideal $\mathcal{P}_{0}$ of $R$. By [12, Exercise 1, p. 41], $\mathcal{P}_{0}=\mathcal{Q}_{0} \bigcap R$, for some $\mathcal{Q}_{0} \in \operatorname{Spec}(T)$. Then, by Lemma $2.1,\left(R / \mathcal{P}_{0}, T / \mathcal{Q}_{0}\right)$ inherits the property of being a survival-pair from $(R, T)$. We claim that no prime ideal of $T / \mathcal{Q}_{0}$ lies over $\mathcal{P} / \mathcal{P}_{0}$. If not, there exists $W \in \operatorname{Spec}(T)$ such that $\mathcal{Q}_{0} \subseteq W$ and $\left(W / \mathcal{Q}_{0}\right) \cap\left(R / \mathcal{P}_{0}\right)=\mathcal{P} / \mathcal{P}_{0}$. More precisely, $\left(W / \mathcal{Q}_{0}\right) \bigcap\left(\left(R+\mathcal{Q}_{0}\right) / \mathcal{Q}_{0}\right)=\left(\mathcal{P}+\mathcal{Q}_{0}\right) / \mathcal{Q}_{0}$. A straightforward calculation reveals that $W \cap R=\mathcal{P}$, contrary to the choice of $\mathcal{P}$. (Indeed, it is clear that $\mathcal{P} \subseteq W \cap R$. For the reverse inclusion, any $w \in W \cap R$ can be written as $w=p+q_{0}$, for some $p \in \mathcal{P}, q_{0} \in \mathcal{Q}_{0}$. Then $q_{0}=w-p \in R+R=R$, whence $q_{0} \in \mathcal{Q}_{0} \bigcap R=\mathcal{P}_{0} \subseteq \mathcal{P}$, so that $w \in \mathcal{P}+\mathcal{P}=\mathcal{P}$, as desired.) This proves the above claim. Therefore, by passing from $(R, T)$ to $\left(R / \mathcal{P}_{0}, T / \mathcal{Q}_{0}\right)$, we may assume, without loss of generality, that $R$ and $T$ are integral domains.

We next reduce to the case in which $\mathcal{P}$ is not lain over by any prime ideal of any subring of $T$ that properly contains $R$. To this end, consider the set

$$
\Gamma:=\{(D, Q) \mid D \text { is a ring, } R \subseteq D \subseteq T, Q \in \operatorname{Spec}(D), Q \bigcap D=\mathcal{P}\}
$$


together with the partial order on $\Gamma$ given by

$$
\left(D_{1}, Q_{1}\right) \leq\left(D_{2}, Q_{2}\right) \Longleftrightarrow\left[D_{1} \subseteq D_{2} \text { and } Q_{2} \bigcap D_{1}=Q_{1}\right] .
$$

As in the proof of [6, Proposition 2.10], Zorn's Lemma applies to $\Gamma$, producing a maximal element $\left(D_{3}, Q_{3}\right)$ of $\Gamma$. Replacing $(R, T, \mathcal{P})$ with $\left(D_{3}, T, Q_{3}\right)$ effects the promised reduction.

In view of the above reduction and the Lying-over Theorem (cf. [12, Theorem 44]) for integral extensions, we see easily that $R$ is integrally closed in $T$. Hence, by Lemma 2.1(b), $U(R)=U(T)$. Next, since $R \subseteq T$ does not satisfy LO, $R \neq T$, and so we can fix $u \in T \backslash R$. By the above reduction, no prime ideal of $R[u]$ lies over $\mathcal{P}$, and so we may replace $T$ with $R[u]$. Thus, without loss of generality, $T=R[u]$. Moreover, $u$ is algebraic over $R$ (since $\mathcal{P} R[X] \in \operatorname{Spec}(R[X]$ ) and $\mathcal{P} R[X] \cap R=\mathcal{P}$ ). It now follows from [12, Exercise 35, p. 44] that $T$ is an overring of $R$.

We next proceed to show that $u^{-1} \in \mathcal{P} R_{\mathcal{P}}$. First, we claim that $1 \in \mathcal{P} R_{\mathcal{P}}[u]$. If not, choose a prime ideal $Q$ of $R_{\mathcal{P}}[u]=R[u]_{R \backslash \mathcal{P}}=T_{R \backslash \mathcal{P}}$ such that $\mathcal{P} R_{\mathcal{P}}[u] \subseteq$ $Q$. Then, necessarily, $Q \cap R_{\mathcal{P}}=\mathcal{P} R_{\mathcal{P}}$, whence $q:=Q \bigcap T \in \operatorname{Spec}(T)$ satisfies $q \bigcap R=\mathcal{P} R_{\mathcal{P}} \cap R=\mathcal{P}$, contrary to the choice of $\mathcal{P}$. This proves the above claim, namely, that $1 \in \mathcal{P} R_{\mathcal{P}}[u]$. In particular, $u$ is a root of a polynomial in $R_{\mathcal{P}}[X]$ having a unit coefficient. Moreover, since $R$ is integrally closed in $T$, we see, as in the proof of [12, Theorem 51], that $R_{\mathcal{P}}$ is integrally closed in $T_{R \backslash \mathcal{P}}$. Thus, by the proof of the $\left(u, u^{-1}\right)$ Lemma [12, Theorem 67], either $u \in R_{\mathcal{P}}$ or $u^{-1} \in R_{\mathcal{P}}$. Notice that the ring $E:=R_{\mathcal{P}} \cap T$ has the property that $R \subseteq E \subseteq T, q^{*}:=\mathcal{P} R_{\mathcal{P}} \cap T \in \operatorname{Spec}(E)$ and $q^{*} \cap R=\mathcal{P}$. Accordingly, by an earlier reduction, $E=R$. As $u \in T \backslash R$, it follows that $u \notin R_{\mathcal{P}}$. Therefore, $u^{-1} \in R_{\mathcal{P}} \backslash U\left(R_{\mathcal{P}}\right)=\mathcal{P} R_{\mathcal{P}}$, as promised. Fix a description $u=a b^{-1}$, with $a \in R \backslash \mathcal{P}$ and $b \in \mathcal{P} \backslash\{0\}$.

Since $R$ is integrally closed in $T=R[u]=R\left[a b^{-1}\right]$, we have that $R$ is also integrally closed in $S:=R[a u]=R\left[a^{2} b^{-1}\right]=R[v]$, where $v:=1+a^{2} b^{-1}$. Observe that the ideal $I:=S v+S b$ of $S$ does not survive in $T$, since $1=$ $v+b\left(-u^{2}\right) \in I T$. Consequently, as (4) ensures that $S \subseteq T$ is a survival extension, $I=S$. Thus

$$
\left(r_{0}+r_{1} v+\cdots+r_{n-1} v^{n-1}\right) v+\left(r_{0}^{*}+r_{1}^{*} v+\cdots+r_{n}^{*} v^{n}\right) b=1
$$

for some elements $r_{i}, r_{j}^{*} \in R$. Defining

$$
t:=\left(r_{0}+b r_{1}^{*}\right)+v\left(r_{1}+b r_{2}^{*}\right)+\cdots+v^{n-1}\left(r_{n-1}+b r_{n}^{*}\right) \in S,
$$

we may rewrite the above equation as $t v+r_{0}^{*} b=1$. Next, observe that $v \neq 0$, for $r_{0}^{*} b \in R b \subseteq \mathcal{P}$. Thus, $z:=t v=1-r_{0}^{*} b \in R$ satisfies $t=z v^{-1} \in R\left[v^{-1}\right]$. Since $t \in S=R[v]$, we can now conclude that $t$ is integral over $R$. (The point is that, being in $R[v] \cap R\left[v^{-1}\right], t$ must be a member of each valuation overring of $R$; one then applies [12, Theorem 57] to the integral closure of $R$.) Therefore, $t \in R$, as $R$ is integrally closed in $T$. 
In fact, $t \in \mathcal{P}$. To see this, use the fact that $\mathcal{P}$ is a prime ideal of $R$, noticing that

$$
t\left(b+a^{2}\right)=b\left[t\left(1+a^{2} b^{-1}\right)\right]=b[t v]=b\left[1-r_{0}^{*} b\right] \in b R \subseteq \mathcal{P},
$$

while $b+a^{2} \in R \backslash \mathcal{P}$. Hence $1=t v+b r_{0}^{*} \in \mathcal{P} S$, contradicting the fact that (4) ensures that $R \subseteq S$ is a survival extension. The proof is complete. $\diamond$

We next infer an enhancement of [6, Corollary 3.6].

Corollary 2.3. Let $R \subseteq T$ be rings. Then $T$ is integral over $R$ if and only if $(R[X], T[X])$ is a survival-pair.

Proof: The "only if" assertion is immediate from the Going-up Theorem for integral extensions (cf. [12, Theorem 44]) and the fact that GU implies survival for extensions. The "if" assertion follows from the implication (4) implies (6) in Theorem 2.2 and the fact [4, Lemma, p. 160] that $T$ is integral over $R$ provided that $R[X] \subseteq T[X]$ satisfies $\mathrm{GU} . \diamond$

As a companion for [6, Proposition 4.5], we next identify an instance of integrality within an arbitrary survival-pair. Following [3], we say that distinct rings $A \subset B$ are adjacent provided that no ring $D$ satisfies $A \subset D \subset B$.

Proposition 2.4. Let $R \subset T$ be distinct rings such that $(R, T)$ is a survivalpair. Then there exist adjacent rings $A \subset B$ such that $R \subseteq A \subset B \subseteq T$ and $B$ is integral over $A$.

Proof: The following proof is inspired by that of [12, Theorem 11]. Using Zorn's Lemma, pick a maximal chain $\left\{S_{\alpha}\right\}$ of rings contained between $R$ and $T$. Fix $t \in T \backslash R$. Put $A:=\bigcup\left\{S_{\alpha} \mid t \notin S_{\alpha}\right\}$ and $B:=\bigcap\left\{S_{\alpha} \mid t \in S_{\alpha}\right\}$. If $t \notin S_{\alpha}$ and $t \in S_{\beta}$, then $S_{\beta} \not \subseteq S_{\alpha}$, whence $S_{\alpha} \subseteq S_{\beta}$. It follows that $A \subseteq B$. In fact, $A \subset B$, since $t \in B \backslash A$. Moreover, $A$ and $B$ are adjacent, for if $D$ were a ring contained properly between $A$ and $B$, then $\left\{S_{\alpha}\right\} \bigcup\{D\}$ would be a chain contradicting the maximality of $\left\{S_{\alpha}\right\}$. Finally, $[3,(2.5 .3)]$ ensures that $A \subseteq B$, being an adjacent survival extension, is necessarily integral. $\diamond$

It is interesting to note that INC-pairs do not satisfy an analogue of Proposition 2.4. Indeed, if $R$ is a valuation domain of Krull dimension 1 and $T$ is the quotient field of $R$, then $(R, T)$ is an INC-pair such that $R \subset T$ is a non-integral extension of adjacent rings.

We pause for some comments about Lemma 2.1.

Remark 2.5. (a) In view of the equivalence of conditions (4) and (5) in Theorem 2.2, we see that Lemma 2.1(a) is a generalization of [6, Lemma 3.1(b)] to the case in which the ideal $J$ is not necessarily prime.

(b) The hypothesis that $R$ is integrally closed in $T$ cannot be deleted from the statement of Lemma 2.1(b). To see this, it suffices to consider any integral extension $R \subseteq T$ such that $U(T) \nsubseteq \subseteq R$, for instance the extension $\mathbb{Z} \subseteq \mathbb{Z}[i]$. 
As is well known, notions such as LO and GU can be generalized from the "extension" context of $[12$, p. 28] to that of homomorphisms. While much carries over to the "homomorphism" context, care is needed as, for instance, [12, Theorem 42] does not generalize. (The point is that GU does not imply LO for homomorphisms. Consider, for example, the canonical surjection $R \longrightarrow R / \mathcal{M}$, where $R$ is a ring of Krull dimension 0 which is not a field, such as $R=\mathbb{Q} \times \mathbb{C}$, and $\mathcal{M}$ is a prime ideal of $R$.) Undaunted, we say that a ring homomorphism $f: R \longrightarrow T$ is a survival homomorphism in case $I T \neq T$ (that is, $f(I) T \neq T$ ) for all proper ideals $I$ of $R$. This notion is characterized in terms of "survival extension" in Proposition 2.6(a); its relation to LO (for homomorphisms) is pursued in Proposition 2.6(b) and Theorem 2.7. A homomorphism-theoretic analogue of Corollary 2.3 is given in Proposition 2.6(c), which depends on the following definition. A ring homomorphism $f: R \longrightarrow T$ is a survival-pair homomorphism in case $A \subseteq B$ is a survival extension for all $R$-subalgebras $A \subseteq B$ of $T$.

Proposition 2.6. Let $f: R \longrightarrow T$ be a ring homomorphism. Then:

(a) $f$ is a survival homomorphism if and only if $f(R) \subseteq T$ is a survival extension such that $k e r(f) \subseteq J(R)$.

(b) If $f$ satisfies $L O$, then $f$ is a survival homomorphism.

(c) $f$ is integral if and only if $f$ is universally a survival-pair homomorphism (in the sense that, for each ring homomorphism $R \longrightarrow S$, the induced homomorphism $S \longrightarrow S \otimes_{R} T$ is a survival-pair homomorphism).

Proof: (a) Put $K:=\operatorname{ker}(f)$. Suppose first that $f$ is a survival homomorphism. Consider a proper ideal $\mathcal{I}$ of $f(R)$. Using the identification $f(R)=R / K$, we have $\mathcal{I}=I / K$ for some proper ideal $I$ of $R$ such that $K \subseteq I$. Observe that $\mathcal{I} T=I T$. The hypothesis on $f$ ensures that $I T \neq T$, whence $\mathcal{I} T \neq T$; that is, $f(R) \subseteq T$ is a survival extension. Now, suppose that $K \nsubseteq \mathcal{M}$ for some maximal ideal $\mathcal{M}$ of $R$. Then $K+\mathcal{M}=R$, so that $a+b=1_{R}$ for some $a \in K, b \in \mathcal{M}$. Then, in $T, 1=f\left(1_{R}\right)=f(a)+f(b)=0+b \cdot 1=b \cdot 1 \in \mathcal{M} T$, contradicting the hypothesis that $\mathcal{M} T \neq T$. Accordingly, no such $\mathcal{M}$ exists; that is, $K \subseteq J(R)$. This completes the proof of the "only if" assertion.

Conversely, suppose that $f(R) \subseteq T$ is a survival extension such that $K \subseteq$ $J(R)$. Our task is to show that if an ideal $I$ of $R$ satisfies $I T=T$, then $I=R$. Once again using the identification $f(R)=R / K$, we have $T=I T=$ $((I+K) / K) T$. As $f(R) \subseteq T$ is a survival extension, it follows that $(I+K) / K=$ $f(R)=R / K$, whence $I+K=R$. Write $c+d=1$, for some $c \in I, d \in K$. Then $I$ contains $c=1-d \in 1+K \subseteq 1+J(R) \subseteq U(R)$, whence $I=R$.

(b) Deny. Then $I T=T$ for some proper ideal $I$ of $R$. Choose $\mathcal{P} \in \operatorname{Spec}(R)$ such that $I \subseteq \mathcal{P}$. As $f$ satisfies LO, there exists $\mathcal{Q} \in \operatorname{Spec}(T)$ such that $f^{-1}(\mathcal{Q})=\mathcal{P}$. Then $f(\mathcal{P}) \subseteq \mathcal{Q}$, whence $T=I T \subseteq \mathcal{P} T=f(\mathcal{P}) T \subseteq \mathcal{Q}$, the desired contradiction.

(c) Suppose first that $f$ is integral. We must prove that $A \subseteq B$ is a survival extension whenever $A \subseteq B$ are $S$-algebras of $S \otimes_{R} T$ arising from a ring homomorphism $R \longrightarrow S$. It suffices to show that $B$ is integral over $A$, and this, in turn, follows since $S \longrightarrow S \otimes_{R} T$ inherits integrality from $f$. 
Conversely, suppose that $f$ is universally a survival-pair homomorphism. Using $S:=R[X]$, we conclude that $R[X] \longrightarrow R[X] \otimes_{R} T=T[X]$ is a survival-pair homomorphism. Thus, $A \subseteq B$ is a survival extension for all $R[X]$-subalgebras $A \subseteq B$ of $T[X]$. In other words, $(f(R)[X], T[X])$ is a survival-pair. Therefore, by Corollary 2.3, $T$ is integral over $f(R)$; equivalently, $f$ is integral, as asserted. $\diamond$

Theorem 2.7. Let $f: R \longrightarrow T$ be a ring homomorphism. Then $f$ satisfies $L O$ if and only if $f$ is universally a survival homomorphism (in the sense that, for each ring homomorphism $R \longrightarrow S$, the induced homomorphism $S \longrightarrow S \otimes_{R} T$ is a survival homomorphism).

Proof: It is well known that LO is a universal property. (This can be shown by, for instance, applying [11, Corollaire 3.2.7.1(i), p. 235].) Thus, if $R \longrightarrow S$ is any ring homomorphism and $f$ satisfies LO, then so does the induced map $S \longrightarrow S \otimes_{R} T$. An application of Proposition 2.6(b) completes the proof of the "only if" assertion.

Conversely, suppose that $f$ is universally a survival homomorphism. Put $K:=\operatorname{ker}(f)$. Suppose the assertion fails. Choose $\mathcal{P} \in \operatorname{Spec}(R)$ such that no $\mathcal{Q} \in \operatorname{Spec}(T)$ satisfies $f^{-1}(\mathcal{Q})=\mathcal{P}$. Consider $I:=f^{-1}(\mathcal{P} T)$, an ideal of $R$ such that $K \subseteq I$ and $\mathcal{P} \subseteq I$. The following analysis involves two cases.

In the first case, $I=\mathcal{P}$. We claim that $I T$ is disjoint from the multiplicatively closed subset $f(R \backslash \mathcal{P})$ of $T$. If not, choose $b \in R \backslash \mathcal{P}$ such that $f(b) \in I T$. Then, by a standard homomorphism theorem,

$$
b \in f^{-1}(I T)=f^{-1}(f(I) T)=f^{-1}(\mathcal{P} T)=I,
$$

so that $b \in I \backslash \mathcal{P}=\emptyset$, a contradiction. This proves the above claim. Therefore, by Zorn's Lemma (cf. [12, p. 2]), there exists $\mathcal{Q} \in \operatorname{Spec}(T)$ such that $I T \subseteq \mathcal{Q}$ and $\mathcal{Q} \cap f(R \backslash \mathcal{P})=\emptyset$. Observe that $\mathcal{P}=I=f^{-1}(f(I)) \subseteq f^{-1}(I T) \subseteq f^{-1}(\mathcal{Q})$. On the other hand, the fact that $\mathcal{Q} \cap f(R \backslash \mathcal{P})=\emptyset$ readily entails that $f^{-1}(\mathcal{Q}) \subseteq \mathcal{P}$. Thus, $f^{-1}(\mathcal{Q})=\mathcal{P}$, the desired contradiction.

In the remaining case, $I \neq \mathcal{P}$. Fix $r \in I \backslash \mathcal{P}$. Applying the hypothesis with $S:=R_{r}$, we have that $R_{r} \longrightarrow R_{r} \otimes_{R} T=: T_{r}$ is a survival homomorphism. (Of course, $T_{r}$ is $R_{r}$-algebra isomorphic to $T_{f(r)}$.) In particular, $1 \notin\left(\mathcal{P} R_{r}\right) T_{r}=\mathcal{P} T_{r}$. However, in $T_{r}$,

$$
1=r r^{-1} \in I T_{r}=f^{-1}(\mathcal{P} T) T_{r} \subseteq(\mathcal{P} T) T_{r}=\mathcal{P} T_{r},
$$

the desired contradiction. The proof is complete. $\diamond$

\section{ACKNOWLEDGEMENT}

The second-named author would like to acknowledge support in part by a University of Tennessee Faculty Development Award and North Dakota State University. Dobbs thanks NDSU for the warm hospitality accorded during his visit in August-September, 2000. 


\section{References}

[1] A. Ayache and A. Jaballah, Residually algebraic pairs of rings, Math. Z. 225 (1997), 49-65.

[2] A. Debremaeker and V. Van Lierde, Integral ring extensions and LO-pairs, Comm. Algebra 28 (2000), 2339-2341.

[3] L. I. Dechéne, Adjacent extensions of rings, dissertation, Univ. California, Riverside (1978).

[4] M. Demazure and P. Gabriel, Introduction to Algebraic Geometry and Algebraic Groups, North Holland, Amsterdam, 1980.

[5] D. E. Dobbs, On INC-extensions and polynomials with unit content, Can. Math. Bull. 23 (1980), 37-42.

[6] D. E. Dobbs, Lying-over pairs of commutative rings, Can. J. Math. 33 (1981), 454-475.

[7] D. E. Dobbs, Nearly integral homomorphisms of commutative rings, Bull. Austral. Math. Soc. 40 (1989), 1-12.

[8] D. E. Dobbs, Prime ideals surviving in complete integral closures, Arch. Math. 69 (1997), 465-469.

[9] M. Fontana, J. A. Huckaba, and I. J. Papick, Prüfer Domains, Monographs and Textbooks Pure Appl. Math. 203, Marcel Dekker, New York, 1997.

[10] R. Gilmer and J. F. Hoffmann, A characterization of Prüfer domains in terms of polynomials, Pac. J. Math. 60 (1975), 81-85.

[11] A. Grothendieck and J. A. Dieudonné, Eléments de Géométrie Algébrique, Springer-Verlag, Berlin, 1971.

[12] I. Kaplansky, Commutative Rings, rev. ed. Univ. Chicago Press, Chicago, 1974.

[13] S. Visweswaran, Intermediate rings between $\mathrm{D}+\mathrm{I}$ and $\mathrm{K}\left[\mathrm{y}_{1}, \ldots, \mathrm{y}_{t}\right]$, Comm. Algebra 18 (1990), 309-345. 